Article

\title{
Riccati Technique and Asymptotic Behavior of Fourth-Order Advanced Differential Equations
}

\author{
Omar Bazighifan 1,2,+®i] and Ioannis Dassios ${ }^{3, *,+}$ \\ 1 Department of Mathematics, Faculty of Science, Hadhramout University, Hadhramout 50512, Yemen; \\ o.bazighifan@gmail.com \\ 2 Department of Mathematics, Faculty of Education, Seiyun University, Hadhramout 50512, Yemen \\ 3 AMPSAS, University College Dublin, D4 Dublin, Ireland \\ * Correspondence: ioannis.dassios@ucd.ie \\ $\dagger$ These authors contributed equally to this work.
}

Received: 9 March 2020; Accepted: 13 April 2020; Published: 15 April 2020

check for updates

Abstract: In this paper, we deal with the oscillation of fourth-order nonlinear advanced differential equations of the form $\left(r(t)\left(y^{\prime \prime \prime}(t)\right)^{\alpha}\right)^{\prime}+p(t) f\left(y^{\prime \prime \prime}(t)\right)+q(t) g(y(\sigma(t)))=0$. We provide oscillation criteria for this type of equations, and examples to illustrate the criteria.

Keywords: oscillatory solutions; fourth-order; advanced differential equations

\section{Introduction}

The present work deals with the investigation of the asymptotic properties of the solutions of fourth-order advanced differential equation of the form

$$
\left(r(t)\left(y^{\prime \prime \prime}(t)\right)^{\alpha}\right)^{\prime}+p(t) f\left(y^{\prime \prime \prime}(t)\right)+q(t) g(y(\sigma(t)))=0,
$$

where $\alpha$ is a quotient of odd positive integers. Throughout this work, we suppose that:

$\left(S_{1}\right) r, p, q \in C\left(\left[t_{0}, \infty\right),[0, \infty)\right), r(t)>0, q>0, r^{\prime}(t)+p(t) \geq 0, \sigma \in C\left(\left[t_{0}, \infty\right),(0, \infty)\right), \sigma(t) \geq t$, $\lim _{t \rightarrow \infty} \sigma(t)=\infty$ and under the condition

$$
\int_{t_{0}}^{\infty}\left[\frac{1}{r(s)} \exp \left(-\int_{t_{0}}^{s} \frac{p(u)}{r(u)} d u\right)\right]^{1 / \alpha} d s<\infty
$$

$\left(S_{2}\right) f, g \in(\mathbb{R}, \mathbb{R}), f(u) \geq k_{f} u^{\alpha}>0, g(u) \geq k_{g} u^{\alpha}>0$ for $u \neq 0$ and $k_{f}, k_{g}$ are constants.

By a solution of (1) we mean a function $y \in C^{\prime \prime \prime}\left[t_{y}, \infty\right), t_{y} \geq t_{0}$, which has the property $r(t)\left(y^{\prime \prime \prime}(t)\right)^{\alpha} \in C^{1}\left[t_{y}, \infty\right)$, and satisfies (1) on $\left[t_{y}, \infty\right)$. We consider only those solutions $y$ of (1) which satisfy $\sup \{|y(t)|: t \geq T\}>0$, for all $T>T_{y}$. We assume that (1) possesses such a solution. A solution of (1) is called oscillatory if it has arbitrarily large zeros on $\left[t_{y}, \infty\right)$, and otherwise it is called to be non-oscillatory. The Equation (1) is said to be oscillatory if all its solutions are oscillatory.

Advanced differential equations have applications in several real- world problems where the evolution rate depends not only on the present, but also on the future. In addition, differential equations in the form of (1) can have application in the mathematical modeling of engineering problems; see [1]. Oscillatory properties of differential equations are fairly well studied by authors in [2-27]. 
Baculikova [28] examined the oscillation of the second-order advanced equation

$$
y^{\prime \prime}(t)+q(t) y(\tau(t))=0 .
$$

She used the generalized Riccati substitution, and established some new sufficient conditions for oscillation. Dzurina [29] established a new comparison principle for advanced canonical equations of the form

$$
\left(r(t) y^{\prime}(t)\right)^{\prime}+q(t) y(\tau(t))=0 .
$$

Many authors, see $[9,13,15,30,31]$ studied the oscillatory behavior of the higher-order advanced differential equation

$$
\left(r(t)\left(y^{(n-1)}(t)\right)^{\alpha}\right)^{\prime}+q(t) y(\tau(t))=0
$$

and established the following results.

Theorem 1. (See [31], Theorem 3.6). Let $\int_{t}^{\infty} q(s) d s<\infty$ such that

$$
\liminf _{t \rightarrow \infty} \int_{t}^{\tau(t)} s^{n-2}\left(\int_{s}^{\infty} q(u) d u\right)^{1 / \alpha} d s>\frac{(n-2) !}{e}
$$

and

$$
\liminf _{t \rightarrow \infty} \int_{t}^{\tau(t)}(\tau(s)-s)^{n-2}\left(\int_{s}^{\infty} q(u) d u\right)^{1 / \alpha} d s>\frac{(n-2) !}{e},
$$

then (3) is oscillatory.

Theorem 2. (See [30]). If for all constants $\theta \in(0,1)$,

$$
\limsup _{t \rightarrow \infty} \frac{t^{n-1}}{(n-1) !}\left(\int_{t}^{\infty} q(s) d s+\frac{\theta \alpha}{2(n-2) !} \int_{t}^{\infty} s^{n-2}\left(\int_{s}^{\infty} q(u) d u\right)^{(\alpha+1) / \alpha} d s\right)^{1 / \alpha}>1,
$$

then (3) is oscillatory.

Theorem 3. (See [13]) Let $\alpha=1$ and $f(y)=y$. If there exists functions $\rho, \vartheta \in C^{1}\left(\left[t_{0}, \infty\right),(0, \infty)\right)$ such that

$$
\int_{t_{0}}^{\infty}\left(\rho(s) q(s) \frac{\mu}{2} \tau^{2}(s)-\frac{1}{4 \rho(s) r(s)}\left[\frac{\rho_{+}^{\prime}(s)}{\rho(s)}-\frac{p(s)}{r(s)}\right]^{2}\right) d s=\infty,
$$

for some $\mu \in(0,1)$, and

$$
\int_{t_{0}}^{\infty}\left[\vartheta(s) \int_{s}^{\infty}\left[\frac{1}{r(v)} \int_{v}^{\infty} q(v)\left(\frac{\sigma^{2}(v)}{v^{2}}\right) d v\right] d v-\frac{\left(\vartheta^{\prime}(s)\right)^{2}}{4 \vartheta(s)}\right] d s=\infty,
$$

then (1) is oscillatory.

Theorem 4. (See [9]) If there exists functions $\delta_{1}, \delta_{2} \in C^{1}\left(\left[t_{0}, \infty\right),(0, \infty)\right)$. Let the equations

$$
y^{\prime}(t)+\delta_{1}(t) y(\sigma(t))=0
$$

and

$$
y^{\prime}(t)+\delta_{2}(t) y(\sigma(t))=0
$$


are oscillatory, then the equation

$$
y^{(4)}(t)+p(t) y^{\prime}(t)+q(t) y(\sigma(t))=0
$$

is oscillatory.

Theorem 5. (See [15]) If there exists function $\rho \in C^{1}\left(\left[t_{0}, \infty\right),(0, \infty)\right)$ such that

$$
\limsup _{t \rightarrow \infty} \frac{1}{H\left(t, t_{0}\right)} \int_{t_{0}}^{\infty}\left(H(t, s) \rho(s) q(s) k(s)-\left(\frac{h(t, s)}{p}\right)^{p} \frac{\rho(s) r(s)}{(H(t, s) G(s) k(s))^{p-1}}\right) d s=\infty,
$$

then the equation

$$
\left(r(t) \Phi\left(y^{(n-1)}(t)\right)\right)^{\prime}+p(t) \Phi\left(y^{(n-1)}(t)\right)+q(t) \Phi(y(g(t)))=0,
$$

where $\Phi=|s|^{p-2} s$ is oscillatory.

1. By applying conditions in Theorem 1, we get

$$
q_{0}>48 /(e \ln 2)
$$

2. By applying conditions in Theorem 2, we get

$$
q_{0}>18
$$

From the above results it can be observed that [31] improves the results in [30]. The motivation in this paper is to complement the results in [15] and improve the results in [30,31] while obtaining some new oscillation criteria for (1).

The paper is organized as follows. In the next section, we will mention some auxiliary lemmas, and in Section 2 we will use the generalized Riccati transformation technique to give some sufficient conditions for the oscillation of (1). In the same section examples are given to illustrate our main results. The method used in this paper is different from that of [15], where they used the integral averaging technique.

Notation 1. For convenience, we denote

$$
\begin{aligned}
\delta\left(t_{0}, t\right) & =\exp \left(\int_{t_{0}}^{t} \frac{p(u)}{r(u)} d u\right) \\
\xi(t) & =\int_{t}^{\infty} \frac{d s}{\left(r(s) \delta\left(t_{0}, s\right)\right)^{\frac{1}{\alpha}}}, \\
\phi(t) & =\frac{\rho^{\prime}(t)}{\rho(t)}-\frac{k_{f} p(t)}{r(t)}, \\
\varphi(t) & =\frac{1}{\delta^{\frac{1}{\alpha}}\left(t_{0}, t\right)}-\frac{\xi(t) p(t) r^{1-\alpha / \alpha}(t)}{\alpha}
\end{aligned}
$$

and

$$
\tilde{\varphi}(t)=\frac{p(t)}{r(t)}+\frac{\alpha^{(\alpha+1)} \rho(t) \varphi^{\alpha+1}(t) \delta\left(t_{0}, t\right)}{\xi(t) r^{\frac{1}{\alpha}}(t)}
$$

\section{Some Auxiliary Lemmas}

We provide the following lemmas: 
Lemma 1. ([32], Lemma 2.2.2) Let $y \in C^{m}\left(\left[t_{0}, \infty\right),(0, \infty)\right)$ such that it and its derivatives up to order $(m-1)$ are absolutely continuous and of constant sign in an interval $\left(t_{0}, \infty\right)$. If $y^{(m-1)}(t) y^{(m)}(t) \leq 0$ for all $t \geq t_{u}, m$ is a positive integer, then for every $\theta \in(0,1)$ there exists a constant $M>0$ such that

$$
y(\theta t) \geq M t^{m-1} y^{(m-1)}(t),
$$

for all sufficient large $t$.

Lemma 2. ([33], Lemma 2.1) Let $\alpha \geq 1$ be a ratio of two odd numbers. $C>0$ and $D$ are constants. Then

$$
D y-C y^{(\alpha+1) / \alpha} \leq \frac{\alpha^{\alpha}}{(\alpha+1)^{\alpha+1}} \frac{D^{\alpha+1}}{C^{\alpha}}, C>0 .
$$

Lemma 3. ([34], Lemma 2.2.3)Let $y \in C^{m}\left(\left[t_{0}, \infty\right),(0, \infty)\right)$. Assume that $y^{(m)}(t)$ is of a fixed sign, on $\left[t_{0}, \infty\right), m$ is a positive integer, $y^{(m)}(t)$ not identically zero and that there exists $a t_{1} \geq t_{0}$ such that for all $t \geq t_{1}$

$$
y^{(m-1)}(t) y^{(m)}(t) \leq 0 .
$$

If we have $\lim _{t \rightarrow \infty} y(t) \neq 0$, then there exists $t_{\lambda} \geq t_{0}$ such that

$$
y(t) \geq \frac{\lambda}{(m-1) !} t^{m-1}\left|y^{(m-1)}(t)\right|
$$

for every $\lambda \in(0,1)$ and $t \geq t_{\lambda}$.

Lemma 4. ([27], Theorem 2.1) Suppose that $y$ is an eventually positive solution of (1). Then, there exist two possible cases:

$$
\begin{aligned}
& \left(\mathbf{N}_{1}\right) y(t)>0, y^{\prime}(t)>0, y^{\prime \prime \prime}(t)>0, y^{(4)}(t)<0 ; \\
& \left(\mathbf{N}_{2}\right) y(t)>0, y^{\prime \prime}(t)>0, y^{\prime \prime \prime}(t)<0 .
\end{aligned}
$$

for $t \geq t_{1}$ where $t_{1} \geq t_{0}$ is sufficiently large.

\section{Oscillation Criteria}

In this section, we establish new oscillation results for Equation (1) by using a generalized Riccati technique. First we prove the following two Lemmas:

Lemma 5. Assume that $y(t)$ is an eventually positive solution of Equation (1) and that $\left(\mathbf{N}_{1}\right)$ holds. If

$$
\psi(t):=\rho(t) \frac{r(t)\left(y^{\prime \prime \prime}\right)^{\alpha}(t)}{y^{\alpha}(t / 2)}
$$

where $\rho \in C^{1}\left(\left[t_{0}, \infty\right),(0, \infty)\right)$ and $M>0$ is constant, then

$$
\psi^{\prime}(t) \leq-k_{g} \rho(t) q(t)+\phi(t) \psi(t)-\frac{\alpha M t^{2}}{2(r(t) \rho(t))^{1 / \alpha}} \psi^{\frac{\alpha+1}{\alpha}}(t) .
$$

Proof. Let $y(t)$ be an eventually positive solution of Equation (1) and from Lemma 4, we see that $\left(\mathbf{N}_{1}\right)$ holds. From Lemma 1, we get

$$
y^{\prime}(t / 2) \geq M t^{2} y^{\prime \prime \prime}(t)
$$


From the definition of $\psi(t)$, we see that $\psi(t)>0$ for $t \geq t_{1}$, and

$$
\begin{aligned}
\psi^{\prime}(t)= & \rho^{\prime}(t) \frac{r(t)\left(y^{\prime \prime \prime}\right)^{\alpha}(t)}{y^{\alpha}(t / 2)}+\rho(t) \frac{\left(r\left(y^{\prime \prime \prime}\right)^{\alpha}\right)^{\prime}(t)}{y^{\alpha}(t / 2)} \\
& -\alpha \rho(t) \frac{y^{\prime}(t / 2) r(t)\left(y^{\prime \prime \prime}\right)^{\alpha}(t)}{2 y^{\alpha+1}(t / 2)} .
\end{aligned}
$$

Using (4) and (6), we obtain

$$
\begin{aligned}
\psi^{\prime}(t) \leq & \frac{\rho^{\prime}(t)}{\rho(t)} \psi(t)+\rho(t) \frac{\left(r\left(y^{\prime \prime \prime}\right)^{\alpha}\right)^{\prime}(t)}{y^{\alpha}(t / 2)} \\
& -\alpha M t^{2} \rho(t) \frac{r(t)\left(y^{\prime \prime \prime}\right)^{\alpha+1}(t)}{2 y^{\alpha+1}(t / 2)}
\end{aligned}
$$

From (1), we get

$$
\begin{aligned}
\psi^{\prime}(t) \leq & \frac{\rho^{\prime}(t)}{\rho(t)} \psi(t)-k_{f} p(t) \frac{\psi(t)}{r(t)} \\
& -k_{g} \rho(t) q(t) \frac{y^{\alpha}(\sigma(t))}{y^{\alpha}(t / 2)}-\alpha M t^{2} \frac{\psi^{\frac{\alpha+1}{\alpha}}(t)}{2(\rho(t) r(t))^{1 / \alpha}} \\
\leq & -k_{g} \rho(t) q(t)+\left(\frac{\rho^{\prime}(t)}{\rho(t)}-k_{f} \frac{p(t)}{r(t)}\right) \psi(t)-\alpha M t^{2} \frac{\psi^{\frac{\alpha+1}{\alpha}}(t)}{2(\rho(t) r(t))^{1 / \alpha}} .
\end{aligned}
$$

Hence, we obtain

$$
\psi^{\prime}(t) \leq-k_{g} \rho(t) q(t)+\phi(t) \psi(t)-\alpha M t^{2} \frac{\psi^{\frac{\alpha+1}{\alpha}}(t)}{2(\rho(t) r(t))^{1 / \alpha}} .
$$

The proof is complete.

Lemma 6. Assume that $y(t)$ is an eventually positive solution of Equation (1) and that $\left(\mathbf{N}_{2}\right)$ holds. Let $k_{g}>1$ be constant. If

$$
\omega(t):=-\frac{r(t)\left(-y^{\prime \prime \prime}\right)^{\alpha}(t)}{\left(y^{\prime \prime}\right)^{\alpha}(t)}
$$

then

$$
\omega^{\prime}(t) \leq \frac{k_{f} p(t)}{r(t) \xi^{\alpha}(t) \delta\left(t_{0}, t\right)}-k_{g} q(t)\left(\frac{\mu}{2} \sigma^{2}(t)\right)^{\alpha}-\alpha \frac{\omega^{\frac{\alpha+1}{\alpha}}(t)}{r^{\frac{1}{\alpha}}(t)}
$$


Proof. Since

$$
\begin{aligned}
\left(-r(t)\left(-y^{\prime \prime \prime}(t)\right)^{\alpha} \delta\left(t_{0}, t\right)\right)^{\prime}= & \left(-r(t)\left(-y^{\prime \prime \prime}(t)\right)^{\alpha}\right)^{\prime} \delta\left(t_{0}, t\right) \\
& +\left(-r(t)\left(-y^{\prime \prime \prime}(t)\right)^{\alpha}\right) \delta\left(t_{0}, t\right) \frac{p(t)}{r(t)} \\
= & (-1)^{\alpha+1}\left(-p(t) f\left(y^{\prime \prime \prime}(t)\right)-q(t) g(y(\sigma(t))) \delta\left(t_{0}, t\right)\right. \\
& -p(t)\left(-y^{\prime \prime \prime}(t)\right)^{\alpha} \delta\left(t_{0}, t\right) \\
\leq & (-1)^{\alpha+1}\left(-k_{f} p(t)\left(y^{\prime \prime \prime}(t)\right)^{\alpha}-k_{g} q(t) y^{\alpha}(\sigma(t))\right) \delta\left(t_{0}, t\right) \\
& -p(t)\left(-y^{\prime \prime \prime}(t)\right)^{\alpha} \delta\left(t_{0}, t\right) \\
= & \left(-p(t)\left(-y^{\prime \prime \prime}(t)\right)^{\alpha}\left(1-k_{f}\right)+k_{g} q(t)\left(-y^{\alpha}(\sigma(t))\right)\right) \delta\left(t_{0}, t\right) \\
= & (-1)^{\alpha}\left(-p(t)\left(y^{\prime \prime \prime}(t)\right)^{\alpha}\left(1-k_{f}\right)+k_{g} q(t)\left(y^{\alpha}(\sigma(t))\right)\right) \delta\left(t_{0}, t\right) \\
\leq & -k_{g} q(t) y^{\alpha}(\sigma(t)) \delta\left(t_{0}, t\right)<0,
\end{aligned}
$$

we conclude that $-r(t)\left(-y^{\prime \prime \prime}(t)\right)^{\alpha} \delta\left(t_{0}, t\right)$ is decreasing. Thus, for $s \geq t \geq t_{1}$

$$
\left(r(s) \delta\left(t_{0}, s\right)\right)^{1 / \alpha} y^{\prime \prime \prime}(s) \leq\left(r(t) \delta\left(t_{0}, t\right)\right)^{1 / \alpha} y^{\prime \prime \prime}(t) .
$$

Dividing both sides of (9) by $\left(r(s) \delta\left(t_{0}, s\right)\right)^{1 / \alpha}$ and integrating the resulting inequality from $t$ to $u$, we get

$$
y^{\prime \prime}(u) \leq y^{\prime \prime}(t)+\left(r(s) \delta\left(t_{0}, s\right)\right)^{1 / \alpha} y^{\prime \prime \prime}(t) \int_{t}^{u} \frac{d s}{\left(r(s) \delta\left(t_{0}, s\right)\right)^{1 / \alpha}} .
$$

Letting $u \rightarrow \infty$, we arrive that

$$
0 \leq y^{\prime \prime}(t)+\left(r(t) \delta\left(t_{0}, t\right)\right)^{1 / \alpha} y^{\prime \prime \prime}(t) \xi(t),
$$

which yields

$$
-\frac{y^{\prime \prime \prime}(t)}{y^{\prime \prime}(t)} \xi(t)\left(r(t) \delta\left(t_{0}, t\right)\right)^{1 / \alpha} \leq 1
$$

Hence,

$$
\frac{r(t)\left(y^{\prime \prime \prime}(t)\right)^{\alpha}}{\left(y^{\prime \prime}(t)\right)^{\alpha}} \geq \frac{-1}{\xi^{\alpha}(t) \delta\left(t_{0}, t\right)} .
$$

From (7), we have

$$
\omega(t) \geq \frac{-1}{\xi^{\alpha}(t) \delta\left(t_{0}, t\right)} .
$$

From the definition of $\omega(t)$, we see that $\omega(t)<0$ for $t \geq t_{1}$, and

$$
\omega^{\prime}(t)=\frac{\left(-r(t)\left(-y^{\prime \prime \prime}(t)\right)^{\alpha}\right)^{\prime}}{\left(y^{\prime \prime}(t)\right)^{\alpha}}-\alpha \frac{-r(t)\left(-y^{\prime \prime \prime}(t)\right)^{\alpha+1}}{\left(y^{\prime \prime}(t)\right)^{\alpha+1}} .
$$

From (1) and (7), we get

$$
\begin{aligned}
\omega^{\prime}(t) & =-k_{f} \frac{p(t)}{r(t)} \omega(t)-k_{g} q(t) \frac{y^{\alpha}(\sigma(t))}{\left(y^{\prime \prime}(t)\right)^{\alpha}}-\alpha \frac{\omega^{\frac{\alpha+1}{\alpha}}(t)}{r^{\frac{1}{\alpha}}(t)} . \\
& =-k_{f} \frac{p(t)}{r(t)} \omega(t)-k_{g} q(t) \frac{y^{\alpha}(\sigma(t))}{\left(y^{\prime \prime}(\sigma(t))\right)^{\alpha}} \frac{\left(y^{\prime \prime}(\sigma(t))\right)^{\alpha}}{\left(y^{\prime \prime}(t)\right)^{\alpha}}-\alpha \frac{\omega^{\frac{\alpha+1}{\alpha}}(t)}{r^{\frac{1}{\alpha}}(t)} .
\end{aligned}
$$


From Lemma 3, we get that for a constant $\mu \in(0,1)$

$$
y(t) \geq \frac{\mu}{2} t^{2} y^{\prime \prime}(t)
$$

Thus, from (10) and (12), we get

$$
\omega^{\prime}(t) \leq \frac{k_{f} p(t)}{r(t) \xi^{\alpha}(t) \delta\left(t_{0}, t\right)}-k_{g} q(t)\left(\frac{\mu}{2} \sigma^{2}(t)\right)^{\alpha}-\alpha \frac{\omega^{\frac{\alpha+1}{\alpha}}(t)}{r^{\frac{1}{\alpha}}(t)}
$$

The proof is complete.

Theorem 6. Assume that (2) holds. Let there exist positive functions $\rho, \vartheta \in C^{1}\left(\left[t_{0}, \infty\right),(0, \infty)\right)$ and $M>0$ be a constant such that

$$
\limsup _{t \rightarrow \infty} \int_{t_{0}}^{t}\left(k_{g} \rho(s) q(s)-\left(\frac{2}{M s^{2}}\right)^{\alpha} \frac{r(s) \rho(s)(\phi(s))^{\alpha+1}}{(\alpha+1)^{\alpha+1}}\right) d s=\infty .
$$

If

$$
\frac{\vartheta(t)}{\xi(t)\left(r(t) \delta\left(t_{0}, t\right)\right)^{1 / \alpha}}+\vartheta^{\prime}(t) \leq 0
$$

and, for $\mu \in(0,1)$,

$$
\limsup _{t \rightarrow \infty} \int_{t_{0}}^{t}\left(k_{g} q(s)\left(\frac{\mu \sigma^{2}(s)}{2} \frac{\vartheta(\sigma(s))}{\vartheta(s)} \xi(s)\right)^{\alpha} \delta\left(t_{0}, s\right)-\tilde{\varphi}(s)\right) d s=\infty,
$$

then every solution of $(1)$ is oscillatory.

Proof. Let $y$ be a non-oscillatory solution of Equation (1) on the interval $\left[t_{0}, \infty\right)$. Without loss of generality, we can assume that $y(t)$ is eventually positive. Using Lemma 4 , we have two cases $\left(\mathbf{N}_{1}\right)$ and $\left(\mathbf{N}_{2}\right)$. For case $\left(\mathbf{N}_{1}\right)$, from Lemma 5, we get that (5) holds. Using Lemma 2, and setting

$$
D=\phi(t), \quad C=\alpha M t^{2} /\left(2(r(t) \rho(t))^{1 / \alpha}\right), \quad y=\psi,
$$

we have:

$$
\psi^{\prime}(t) \leq-k_{g} \rho(t) q(t)+\left(\frac{2}{M t^{2}}\right)^{\alpha} \frac{r(t) \rho(t)(\phi(t))^{\alpha+1}}{(\alpha+1)^{\alpha+1}} .
$$

Integrating from $t_{1}$ to $t$, we get:

$$
\int_{t_{1}}^{t}\left(k_{g} \rho(s) q(s)-\left(\frac{2}{M s^{2}}\right)^{\alpha} \frac{r(s) \rho(s)(\phi(s))^{\alpha+1}}{(\alpha+1)^{\alpha+1}}\right) d s \leq \psi\left(t_{1}\right),
$$

which contradicts (13). For the case $\left(\mathbf{N}_{2}\right)$, from the proof of Lemma 6, we have:

$$
\frac{y^{\prime \prime \prime}(t)}{y^{\prime \prime}(t)} \geq \frac{-1}{\xi(t)\left(r(t) \delta\left(t_{0}, t\right)\right)^{1 / \alpha}}
$$


Using the latter inequality and (14), we obtain

$$
\begin{aligned}
\left(\frac{y^{\prime \prime}(t)}{\vartheta(t)}\right)^{\prime} & =\frac{y^{\prime \prime \prime}(t) \vartheta(t)-y^{\prime \prime}(t) \vartheta \prime(t)}{\vartheta^{2}(t)} \\
& \geq \frac{y^{\prime \prime}(t)}{\vartheta^{2}(t)}\left(\frac{\vartheta(t)}{\xi(t)\left(r(t) \delta\left(t_{0}, t\right)\right)^{1 / \alpha}}+\vartheta^{\prime}(t)\right) \geq 0
\end{aligned}
$$

which implies that $y^{\prime \prime}(t) / \vartheta(t)$ is nondecreasing. Hence, it follows from $\sigma(t) \geq t$ that

$$
\frac{y^{\prime \prime}(\sigma(t))}{y^{\prime \prime}(t)} \geq \frac{\vartheta(\sigma(t))}{\vartheta(t)}
$$

Thus, by using (11) and (12), we get

$$
\omega^{\prime}(t) \leq \frac{k_{f} p(t)}{r(t) \xi^{\alpha}(t) \delta\left(t_{0}, t\right)}-k_{g} q(t)\left(\frac{\mu}{2} \sigma^{2}(t)\right)^{\alpha}\left(\frac{\vartheta(\sigma(t))}{\vartheta(t)}\right)^{\alpha}-\alpha \frac{\omega^{\frac{\alpha+1}{\alpha}}(t)}{r^{\frac{1}{\alpha}}(t)} .
$$

Multiplying (17) by $\xi^{\alpha}(t) \delta\left(t_{0}, t\right)$ and integrating from $t_{1}$ to $t$, we get

$$
\begin{aligned}
& \xi^{\alpha}(t) \delta\left(t_{0}, t\right) \omega(t)-\xi^{\alpha}\left(t_{1}\right) \delta\left(t_{0}, t_{1}\right) \omega\left(t_{1}\right)-\int_{t_{1}}^{t} \frac{p(s)}{r(s)} d s \\
& +\alpha \int_{t_{1}}^{t} r^{\frac{-1}{\alpha}}(s) \xi^{\alpha-1}(s) \delta\left(t_{0}, s\right) \varphi(s) \omega(s) d s \\
& +\int_{t_{1}}^{t} k_{g} q(s)\left(\frac{\mu}{2} \sigma^{2}(s)\right)^{\alpha}\left(\frac{\vartheta(\sigma(s))}{\vartheta(s)}\right)^{\alpha} \xi^{\alpha}(s) \delta\left(t_{0}, s\right) d s \\
& +\alpha \int_{t_{1}}^{t} \frac{\omega^{\frac{\alpha+1}{\alpha}}(s)}{r^{\frac{1}{\alpha}}(s)} \xi^{\alpha}(s) \delta\left(t_{0}, s\right) d s \\
& \leq 0 .
\end{aligned}
$$

Using Lemma 2 we set

$$
C=\xi^{\alpha}(s) \delta\left(t_{0}, s\right) / r^{\frac{1}{\alpha}}(s), D=\int_{t_{1}}^{t} r^{\frac{-1}{\alpha}}(s) \xi^{\alpha-1}(s) \delta\left(t_{0}, s\right) \varphi(s), y=\omega(t) .
$$

Thus, we get

$$
\begin{aligned}
& \xi^{\alpha}(t) \delta\left(t_{0}, t\right) \omega(t)-\xi^{\alpha}\left(t_{1}\right) \delta\left(t_{0}, t_{1}\right) \omega\left(t_{1}\right)-\int_{t_{1}}^{t} \frac{p(s)}{r(s)} d s \\
& +\int_{t_{1}}^{t} k_{g} q(s)\left(\frac{\mu}{2} \sigma^{2}(s)\right)^{\alpha}\left(\frac{\vartheta(\sigma(s))}{\vartheta(s)}\right)^{\alpha} \xi^{\alpha}(s) \delta\left(t_{0}, s\right) d s \\
& +\int_{t_{1}}^{t} \frac{\alpha^{(\alpha+1)} \rho(s) \varphi^{\alpha+1}(s) \delta\left(t_{0}, s\right)}{\xi(s) r^{\frac{1}{\alpha}}(t)} d s \\
& \leq \quad 0 .
\end{aligned}
$$

Hence, by using (10), we obtain

$$
\int_{t_{1}}^{t}\left(k_{g} q(s)\left(\frac{\mu \sigma^{2}(s)}{2} \frac{\vartheta(\sigma(s))}{\vartheta(s)} \xi(s)\right)^{\alpha} \delta\left(t_{0}, s\right)-\tilde{\varphi}(s)\right) d s \leq \xi^{\alpha}(t) \delta\left(t_{0}, t\right) \omega\left(t_{1}\right)+1,
$$

which contradicts (15). Theorem 6 is proved. 
If we define the function

$$
\omega(t):=\vartheta(t) \frac{y^{\prime}(t)}{y(t)}
$$

by using it into (1), we prove the following corollary:

Corollary 1. Assume that (2) holds. If there exist positive functions $\rho, \vartheta \in C^{1}\left(\left[t_{0}, \infty\right),(0, \infty)\right)$ such that (14) is satisfied and

$$
\begin{gathered}
\limsup _{t \rightarrow \infty} \int_{t_{0}}^{t}\left(k_{g} \rho(s) q(s)-\frac{r(s) \rho(s)(\phi(s))^{\alpha+1}}{(\alpha+1)^{\alpha+1}}\right) d s=\infty, \\
\limsup _{t \rightarrow \infty} \int_{t_{0}}^{t}\left(k_{g} q(s)\left(\frac{\vartheta(\sigma(s))}{\vartheta(s)} \xi(s)\right)^{\alpha} \delta\left(t_{0}, s\right)-\tilde{\varphi}(s)\right) d s=\infty,
\end{gathered}
$$

then every solution of (1) is oscillatory.

Remark 1. By using similar methods to [22,23], we can easily establish Philos-type, Hille and Nehari-type oscillation criteria for (1).

Example 1. Consider the differential equation

$$
\left(t^{2}\left(y^{\prime \prime \prime}(t)\right)\right)^{\prime}+\frac{t}{2} y^{\prime \prime \prime}(t)+\frac{q_{0}}{t^{2}} y(2 t)=0,
$$

where $q_{0}>0$ is constant. Please note that $\alpha=1, t_{0}=1, r(t)=t^{2}, p(t)=t / 2, q(t)=t, \sigma(t)=2 t$. We now set $\rho(t)=t, k_{f}=k_{g}=1$, then:

$$
\begin{aligned}
\delta\left(t_{0}, t\right) & =\exp \left(\int_{t_{0}}^{t} \frac{p(u)}{r(u)} d u\right)=t^{1 / 2}, \xi(t)=\int_{t}^{\infty} \frac{d s}{\left(r(s) \delta\left(t_{0}, s\right)\right)^{\frac{1}{\alpha}}}=\frac{2 t^{-3 / 2}}{3} \\
\vartheta(t) & =\frac{2 t^{-3 / 2}}{3}, \varphi(t)=\frac{1}{\delta^{\frac{1}{\alpha}}\left(t_{0}, t\right)}-\frac{\xi(t) p(t) r^{1-\alpha / \alpha}(t)}{\alpha}=\frac{2 t^{-1 / 2}}{3}, \phi(t)=\frac{-1}{2 t}
\end{aligned}
$$

and

$$
\tilde{\varphi}(t)=\frac{p(t)}{r(t)}+\frac{\alpha^{(\alpha+1)} \rho(t) \varphi^{\alpha+1}(t) \delta\left(t_{0}, t\right)}{\xi(t) r^{\frac{1}{\alpha}}(t)}=\frac{2 t^{-1 / 3}}{3} .
$$

Thus, we get

$$
\limsup _{t \rightarrow \infty} \int_{t_{0}}^{t}\left(k_{g} \rho(s) q(s)-\left(\frac{2}{M s^{2}}\right)^{\alpha} \frac{r(s) \rho(s)(\phi(s))^{\alpha+1}}{(\alpha+1)^{\alpha+1}}\right) d s=\infty
$$

and

$$
\frac{\vartheta(t)}{\xi(t)\left(r(t) \delta\left(t_{0}, t\right)\right)^{1 / \alpha}}+\vartheta^{\prime}(t)=0
$$

Furthermore, for $\mu \in(0,1)$,

$$
\limsup _{t \rightarrow \infty} \int_{t_{0}}^{t}\left(k_{g} q(s)\left(\frac{\mu \sigma^{2}(s)}{2} \frac{\vartheta(\sigma(s))}{\vartheta(s)} \xi(s)\right)^{\alpha} \delta\left(t_{0}, s\right)-\tilde{\varphi}(s)\right) d s=\infty .
$$

Hence, by using Theorem 6, every solution of Equation (20) is oscillatory.

Example 2. Consider the differential equation

$$
\left(t^{2}\left(y^{\prime}(t)\right)\right)^{\prime}+\frac{t}{2} y^{\prime}(t)+q_{0} y(2 t)=0
$$


where $q_{0}>0$ is constant. Please note that $\alpha=1, t_{0}=1, r(t)=t^{2}, p(t)=t / 2, q(t)=q_{0}, \sigma(t)=2 t$. We now set $\rho(t)=k_{f}=k_{g}=1$, then $\xi(t)=\vartheta(t)=2 t^{-3 / 2} / 3$.

It is easy to observe that condition (18) holds and that condition (19) is satisfied for $q_{0}>2 \sqrt{2}$. Hence, by using Corollary 1, every solution of Equation (21) is oscillatory if $q_{0}>2 \sqrt{2}$.

\section{Conclusions}

In this paper, we provided new oscillation criteria for (1) by using a Riccati transformationtechnique. As a further extension of this article, one can consider the case of $z(t)=y(t)+p(t) y((\sigma(t)))$, and try to get some oscillation criteria of (1) by using a method of comparison with first-order delay equations and the integral averaging technique.

Author Contributions: Writing original draft, writing review and editing, O.B.; Formal analysis, writing review and editing, funding and supervision, I.D. All authors have read and agreed to the published version of the manuscript.

Funding: This work was supported by Science Foundation Ireland (SFI), by funding Ioannis Dassios, under Investigator Programme Grant No. SFI/15 /IA/3074.

Acknowledgments: The authors thank the reviewers for their useful comments, which led to the improvement of the content of the paper.

Conflicts of Interest: There are no competing interests between the authors.

\section{References}

1. Hale, J.K. Theory of Functional Differential Equations; Springer-Verlag: New York, NY, USA, 1977.

2. Bazighifan, O.; Cesarano, C. Some New Oscillation Criteria for Second-Order Neutral Differential Equations with Delayed Arguments. Mathematics 2019, 7, 619. [CrossRef]

3. Bazighifan, O.; Elabbasy, E.M.; Moaaz, O. Oscillation of higher-order differential equations with distributed delay. J. Inequal. Appl. 2019, 2019, 55. [CrossRef]

4. Chatzarakis, G.E.; Elabbasy, E.M.; Bazighifan, O. An oscillation criterion in 4th-order neutral differential equations with a continuously distributed delay. Adv. Differ. Equ. 2019, 2019, 336. [CrossRef]

5. Cesarano, C.; Pinelas, S.; Al-Showaikh, F.; Bazighifan, O. Asymptotic Properties of Solutions of Fourth-Order Delay Differential Equations. Symmetry 2019, 11, 628. [CrossRef]

6. Cesarano, C.; Bazighifan, O. Oscillation of fourth-order functional differential equations with distributed delay. Axioms 2019, 7, 61. [CrossRef]

7. Cesarano, C.; Bazighifan, O. Qualitative behavior of solutions of second order differential equations. Symmetry 2019, 11, 777. [CrossRef]

8. Chatzarakis, G.E.; Li, T. Oscillation criteria for delay and advanced differential equations with nonmonotone arguments. Complexity 2018, 2018, 8237634. [CrossRef]

9. Dzurina, J; Jadlovska, I. Oscillation theorems for fourth order delay differential equations with a negative middle term. Meth. Appl. Sci. 2017, 6, 1-13.

10. El-Nabulsi, R.A.; Moaaz, O.; Bazighifan, O. New Results for Oscillatory Behavior of Fourth-Order Differential Equations, Symmetry 2020, 12, 136. [CrossRef]

11. Elabbasy, E.M.; Cesarano, C.; Bazighifan, O.; Moaaz, O. Asymptotic and oscillatory behavior of solutions of a class of higher order differential equation. Symmetry 2019, 11, 1434. [CrossRef]

12. Bazighifan, O.; Cesarano, C. A Philos-Type Oscillation Criteria for Fourth-Order Neutral Differential Equations. Symmetry 2020, 12, 379. [CrossRef]

13. Elabbasy, E.M.; Thandpani, E.; Moaaz, O.; Bazighifan, O. Oscillation of solutions to fourth-order delay differential equations with midlle term. Open J. Math. Sci. 2019, 3, 191-197. [CrossRef]

14. Li, T.; Han, Z.; Zhao, P.; Sun, S. Oscillation of even-order neutral delay differential equations. Adv. Differ. Equ. 2010, 2010, 184180. [CrossRef]

15. Liu, S.; Zhang, Q.; Yu, Y. Oscillation of even-order half-linear functional differential equations with damping Compu. Math. Appl. 2010, 61, 2191-2196.

16. Kiguradze, I.T.; Chanturiya, T.A. Asymptotic Properties of Solutions of Nonautonomous Ordinary Differential Equations; Kluwer Acad. Publ.: Dordrecht, The Netherlands, 1993. 
17. Moaaz, O.; Kumam, P.; Bazighifan, O. On the Oscillatory Behavior of a Class of Fourth-Order Nonlinear Differential Equation. Symmetry 2020, 12, 524. [CrossRef]

18. Moaaz, O.; Dassios, I.; Bazighifan, O.; Muhib, A. Oscillation Theorems for Nonlinear Differential Equations of Fourth-Order. Mathematics 2020, 8, 520. [CrossRef]

19. Moaaz, O.; Dassios, I.; Bazighifan, O. Oscillation Criteria of Higher-order Neutral Differential Equations with Several Deviating Arguments. Mathematics 2020, 8, 412. [CrossRef]

20. Moaaz, O.; Elabbasy, E.M.; Muhib, A. Oscillation criteria for even-order neutral differential equations with distributed deviating arguments. Adv. Differ. Equ. 2019, 2019, 297. [CrossRef]

21. Bazighifan, O. An Approach for Studying Asymptotic Properties of Solutions of Neutral Differential Equations. Symmetry 2020, 12, 555. [CrossRef]

22. Nehari, Z. Oscillation criteria for second order linear differential equations. Trans. Am. Math. Soc. 1957, 85, 428-445. [CrossRef]

23. Philos, $\mathrm{C}$. On the existence of nonoscillatory solutions tending to zero at $\infty$ for differential equations with positive delay. Arch. Math. 1981, 36, 168-178. [CrossRef]

24. Park, C.; Moaaz, O.; Bazighifan, O. Oscillation Results for Higher Order Differential Equations. Axioms 2020, 9, 14. [CrossRef]

25. Xing, G.; Li, T.; Zhang, C. Oscillation of higher-order quasi linear neutral differential equations. Adv. Differ. Equ. 2011, 2011, 45. [CrossRef]

26. Zafer, A. Oscillation criteria for even order neutral differential equations. Appl. Math. Lett. 1998, 11, $21-25$. [CrossRef]

27. Zhang, C.; Li, T.; Sun, B.; Thandapani, E. On the oscillation of higher-order half-linear delay differential equations. Appl. Math. Lett. 2011, 24, 1618-1621. [CrossRef]

28. Baculikova, B. Oscillatory behavior of the second order functional differential equations. Appl. Math. Lett. 2017, 72, 35-41. [CrossRef]

29. Dzurina, J. A comparison theorem for linear delay differential equations. Arch. Math. Brno 1995, 31, 113-120.

30. Agarwal, R.; Grace, S.R.; O'Regan, D. Oscillation criteria for certain $\mathrm{n}$ th order differential equations with deviating arguments. J. Math. Anal. Appl. 2001, 262, 601-622. [CrossRef]

31. Agarwal, R.; Grace, S.R. Oscillation theorems for certain functional differential equations of higher order. Math. Comput. Model. 2004, 39, 1185-1194. [CrossRef]

32. Agarwal, R.; Grace, S.; O'Regan, D. Oscillation Theory for Difference and Functional Differential Equations; Kluwer Acad. Publ.: Dordrecht, The Netherlands, 2000.

33. Agarwal, R.P.; Zhang, C.; Li, T. Some remarks on oscillation of second order neutral differential equations. Appl. Math. Comput.2016, 274, 178-181. [CrossRef]

34. Bazighifan, O.; Postolache, M. An improved conditions for oscillation of functional nonlinear differential equations. Mathematics 2020, 8, 552. [CrossRef] 